Wilson Kioshi Matsumoto 1

Maria Glória Vicente 2

Maria Aparecida Silva 2

Lia Lusitana Cardozo de Castro 1

\section{Comportamento epidemiológico da malária nos municípios que compõem a Bacia do Alto Paraguai, Mato Grosso do Sul, no período de 1990 a 1996}

\author{
Epidemiological trends for malaria \\ in the Upper Paraguay River Basin, \\ Mato Grosso do Sul, Brazil 1990-1996
}

1 Superintendência de Desenvolvimento de Recursos Humanos, Secretaria de Estado de Saúde do Mato Grosso do Sul. Av. Senador Filinto Müller 1480, Campo Grande, MS 79004-480, Brasil. 2 Centro de Informações de Medicamentos,

Departamento de Tecnologia de Alimentos e Saúde Pública, Centro de Ciências Biológicas e da Saúde, Universidade Federal do Mato Grosso do Sul. C. P. 649 , Campo Grande, MS 79070-900, Brasil.
Abstract Through the Regional Office of the Brazilian National Health Foundation in the State of Mato Grosso do Sul, we obtained numerical data on malaria for the Upper Paraguay Basin (UPB): 159 cases in 1990, 126 in 1991, 135 in 1992, 61 in 1993, 143 in 1994, 41 in 1995, and 20 in 1996, the majority of which were imported cases. There were no autochthonous cases in 1990, and since 1991 the rates of over 15\% dropped to around $1.60 \%$. Imported cases, corresponding to $0.63 \%$ in 1990, increased in 1991 and 1992 to some $1.50 \%$, and to 3.28\% in 1993. Induced cases were recorded only in 1991 and 1992 (less than 1\%). Most cases were between 16 and 45 years of age. There was a predominance of Plasmodium vivax in the thick blood smears. Although autochthonous cases of malaria are not the majority, the disease is still an important public heal th problem in the UPB in the presence of the Anopheles (N.) darlingi vector and human migration into the region.

Key words Anopheles; Plasmodium vivax; Malaria; Epidemiology

Resumo Com base em dados secundários levantados na Coordenação Regi onal da Fundação Nacional de Saúde/Ministério da Saúde (FNS/MS), obteve-se o número de casos de malária registrados na Bacia do Alto Paraguai (BAP), no Mato Grosso do Sul (MS): 159, 126, 135, 61, 143, 41 e 20 em, respectivamente, 1990, 1991, 1992, 1993, 1994, 1995 e 1996, sendo a grande maioria destes de casos importados. Em 1990 não houve casos autóctones, ea partir de 1991 os índices superiores a $15 \%$ foram diminuindo até ficar próximo de 1,60\%. Os casos introduzi dos, que 1990 correspondiam a 0,63\%, aumentaram em 1991 e 1992, mantendo-se em torno de 1,50\%, atingindo em 1993 percentuais de 3,28\%. Foram registrados casos induzi dos somente em 1991 e 1992 (menos de 1\%). A faixa etária mais atingi da foi a compreendi da entre 16 e 45 anos. Houve um predomínio do Plasmodium vivax no total de lâminas exami nadas. Embora os casos autóctones de malária não sejam mai oria, a doença constitui importante problema de Saúde Pública na BAP em razão da presença do vetor Anopheles (N.) darlingi e das correntes migratórias para a região. Palavras-chave Anopheles; Plasmodium vivax; Malária; Epidemiologia 


\section{Introdução}

A malária, também conhecida como impaludismo, febre palustre, sezão e maleita, é uma das enfermidades parasitárias mais importantes entre as que afetam as populações dos países tropicais e subtropicais do mundo.

Os sinais e sintomas clínicos da malária são a febre, o acesso malárico (calafrio, calor e suor) e a anemia, além de sintomas gerais, como cefaléia, mialgia, mal-estar, indisposição e, nos casos graves, comprometimento visceral (esplenomegalia e hepatomegalia) (Veronezi, 1983).

$\mathrm{O}$ agente etiológico da doença foi identificado pela primeira vez em 1880 por Charles Alphonse Laveran. A patologia é causada por espécies de protozoários do gênero Plasmodium: P. vivax, responsável pela doença terçã benigna; P. falci parum, agente da doença terçã maligna; P. malariae, causador da doença quartã benigna; e P. ovale, também causador da doença terçã benigna, que existe apenas na África. A transmissão se faz pela picada da fêmea de mosquitos infectados pelo parasito do gênero Anopheles (Sucen, 1988).

Atualmente, ocorrem de trezentos a quinhentos milhões de casos no mundo, causando dois milhões de óbitos, principalmente em crianças menores de dois anos (MS, 1996b).

No Brasil, a doença acomete anualmente mais de 560.000 indivíduos, numa população total de 154 milhões de habitantes, com um índice de positividade anual de 3,7 por mil habitantes (Opas, 1991).

Em Mato Grosso do Sul, a Bacia do Alto Paraguai (BAP), é composta por 28 municípios, agrupados em cinco microrregiões, a saber: Baixo Pantanal, Aquidauana, Campo Grande, Bodoquena e Alto Taquari (UFMS, 1996). Em face da ocupação irracional das cabeceiras dos rios pelo homem, a região encontra-se ameaçada de desequilíbrio de ordens ambiental e sanitária, o que reclama estudos que propiciem estruturação de um sistema de planejamento para a BAP.

Visando incorporar indicadores da qualidade de vida da população aos estudos de planejamento da BAP, propomo-nos a estudar a malária, pois, embora os casos de malária não sejam autóctones em sua maioria, a doença constitui importante problema de saúde pública na região. Tal fato se dá por causa da presença do vetor e das correntes migratórias decorrentes da vocação turística de alguns municípios, bem como pela presença de garimpos e da demanda de mão-de-obra oriunda de certas partes do Brasil e de países vizinhos (Paraguai e
Bolívia), apontando assim para a necessidade de estudos que contribuam para o controle dessa enfermidade e para a conservação do meio ambiente.

Nesse trabalho, procurou-se conhecer o comportamento epidemiológico da malária nos municípios que compõem a Bacia do Alto Paraguai, Mato Grosso do Sul (BAP/MS), no período de 1990 a 1996, por meio da identificação das microrregiões da BAP que apresentaram casos de malária, do levantamento do grupo etário de maior ocorrência da patologia, da identificação das espécies de Plasmodium responsáveis pelos casos de malária e da apresentação e classificação dos casos notificados segundo a origem de infecção.

Ressaltamos a inviabilidade de se calcular o coeficiente de incidência segundo as faixas etárias que o Serviço de Epidemiologia e Operações da Coordenação Regional da Fundação Nacional de Saúde/ Mato Grosso do Sul utiliza para condensar os casos notificados, pois os dados populacionais disponíveis não contemplam esses grupos etários.

\section{Metodologia}

O presente trabalho é um estudo descritivo da situação epidemiológica da malária nos municípios que compõem a Bacia do Alto Paraguai/ Mato Grosso do Sul (BAP/MS), no período de 1990 a 1996. Para a elaboração da pesquisa, foram utilizados dados secundários de morbidade da Coordenação Regional da Fundação Nacional de Saúde/ Mato Grosso do Sul, provenientes das fichas de investigação de casos e de tratamento radical. Os dados populacionais foram obtidos na Divisão de Informação à Saúde da Secretaria de Estado de Saúde de Mato Grosso do Sul, sendo os mesmos ajustados para lo de julho.

Utilizou-se a definição de caso proposto pelo Ministério da Saúde (MS, 1994c), conforme segue:

- Suspeito: todo indivíduo que, procedendo da área onde haja transmissão da malária ou tendo recebido transfusão sangüínea, apresenta um quadro febril, especialmente se for acompanhado por sintomas sugestivos de malária.

- Confirmado: caso com confirmação laboratorial indicando a presença de parasitos no sangue. É importante e necessária a identificação da espécie.

A classificação de casos de malária é feita de acordo com a origem da infecção (MS, 1995b):

- Caso autóctone: caso de malária contraída pelo enfermo na zona de sua residência. 
- Caso importado: caso de malária contraída fora da zona onde se fez o diagnóstico.

- Caso induzido: caso de malária que pode ser atribuído a uma transfusão de sangue ou outra forma de inoculação parenteral, e não à transmissão natural pelo mosquito.

- Caso introduzido: casos secundários diretos, quando se pode provar que os mesmos constituem o primeiro elo de transmissão local após um caso importado conhecido.

\section{Resultados e discussões}

No período de 1990 a 1996, foram notificados 3.544 casos de malária no Mato Grosso do Sul, sendo 685 deste total procedentes dos municípios da Bacia do Alto Paraguai, Mato Grosso do Sul (BAP/MS).

A Figura 1 demonstra que ocorreu declínio do coeficiente de incidência na BAP/MS no período de estudo, apresentando, porém, um pico em 1994 e voltando a cair nos anos subseqüentes. A curva de incidência refletiu a situação da doença no Estado, exceto em 1994, quando o pico foi observado somente na Bacia do Alto Paraguai/MS, provavelmente em razão do grande número de casos registrados no Município de Bonito ( $n=93 /$ coeficiente de incidên$\mathrm{cia}=548,20 / 100.000$ habitantes). Segundo jornais da época e informações verbais dos próprios técnicos da Coordenação Regional da Fundação Nacional de Saúde em Mato Grosso do Sul (COOR-FNS/MS), esses casos ocorreram após um fluxo migratório motivado pala notícia da existência de metais preciosos na região.

Das cinco microrregiões, a do Alto Taquari foi a que registrou os maiores coeficientes de incidência no período, exceto em 1991, quando a de Campo Grande apresentou coeficiente de 77,47/ 100.000 habitantes (Figura 2). O Município de Rochedo foi responsável por 22 $(68,75 \%)$ dos 32 casos registrados na microrregião de Campo Grande em 1991, traduzindo-se num coeficiente de incidência de 625,71/ 100.000 habitantes. Rochedo é um município onde normalmente o fluxo de pessoas é baixo, entretanto, em fins de 1990, correntes migratórias procedentes da região Norte vieram em busca de trabalho, em face da contratação de diaristas para as plantações de arroz. Outro fator que pode ter contribuído para este fluxo de pessoas é a existência de um pesqueiro que atrai turistas para a região (Kanomata, 1994).

O grupo etário que concentrou mais de $70 \%$ dos casos foi o de 16 a 45 anos, particularmente o grupo de 16 a 30 anos, conforme mostra o Figura 3.
Figura 1

Malária - coeficiente de incidência/100.000 habitantes na Bacia do Alto Paraguai/ Mato Grosso do Sul, no período de 1990 a 1996.

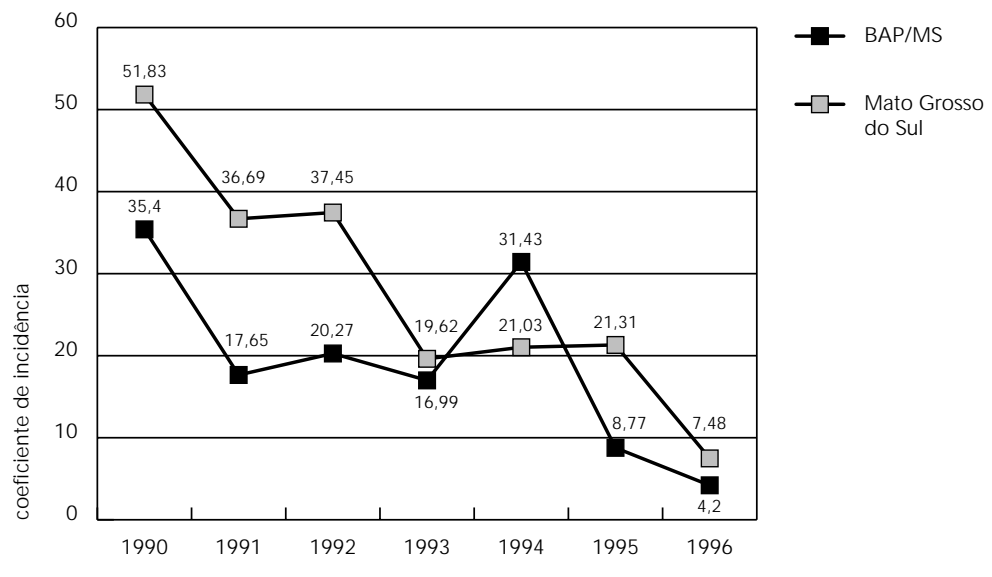

Fonte: MS/FNS/CO OR-MS/SEOPE, 1990-1997.

Figura 2

Malária - coeficiente de incidência/100.000 habitantes na Bacia do Alto Paraguai/ Mato Grosso do Sul, segundo a microrregião, no período de 1990 a 1996.

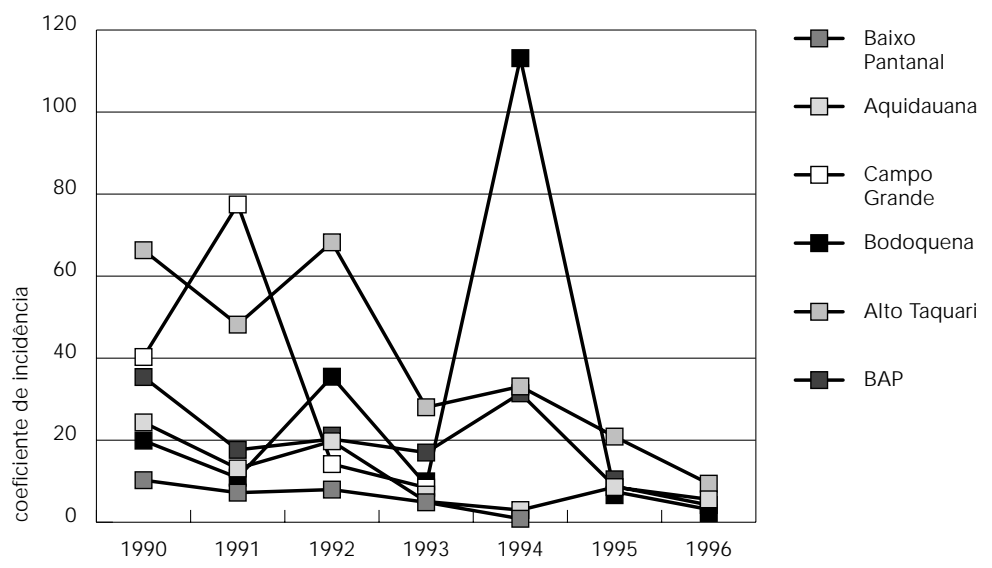

Fonte: MS/FNS/CO OR-MS/SEOPE, 1990-1997.

Em Mato Grosso do Sul, o diagnóstico é feito empregando-se o método de Walker, com a coleta do material (sangue) durante ou após o acesso malárico (Sucen, 1988).

O diagnóstico clínico é feito através da anamnese, a qual evidencia os sinais e sintomas da patologia. No entanto, para uso de te- 
rapêutica eficaz, é fundamental, entre outras coisas, determinar a espécie do Plasmodium, o que somente é possível através do exame laboratorial (MS, 1994b).

Segundo os dados do Figura 4, os casos de malária registrados na Bacia do Alto Paraguai são predominantemente causados pelo Plas- modium vivax, situação que reflete o comportamento da doença em Mato Grosso do Sul, que também registrou a maioria dos casos por esta espécie (MS, 1996b).

No âmbito nacional, no período de 1990 a 1993, houve predomínio do Plasmodium vivax (Marques et al., 1994). O Plasmodium fal cipa-

Figura 3

Malária - casos registrados na Bacia do Alto Paraguai/Mato Grosso do Sul, segundo o grupo etário e ano, no período de 1990 a 1996.

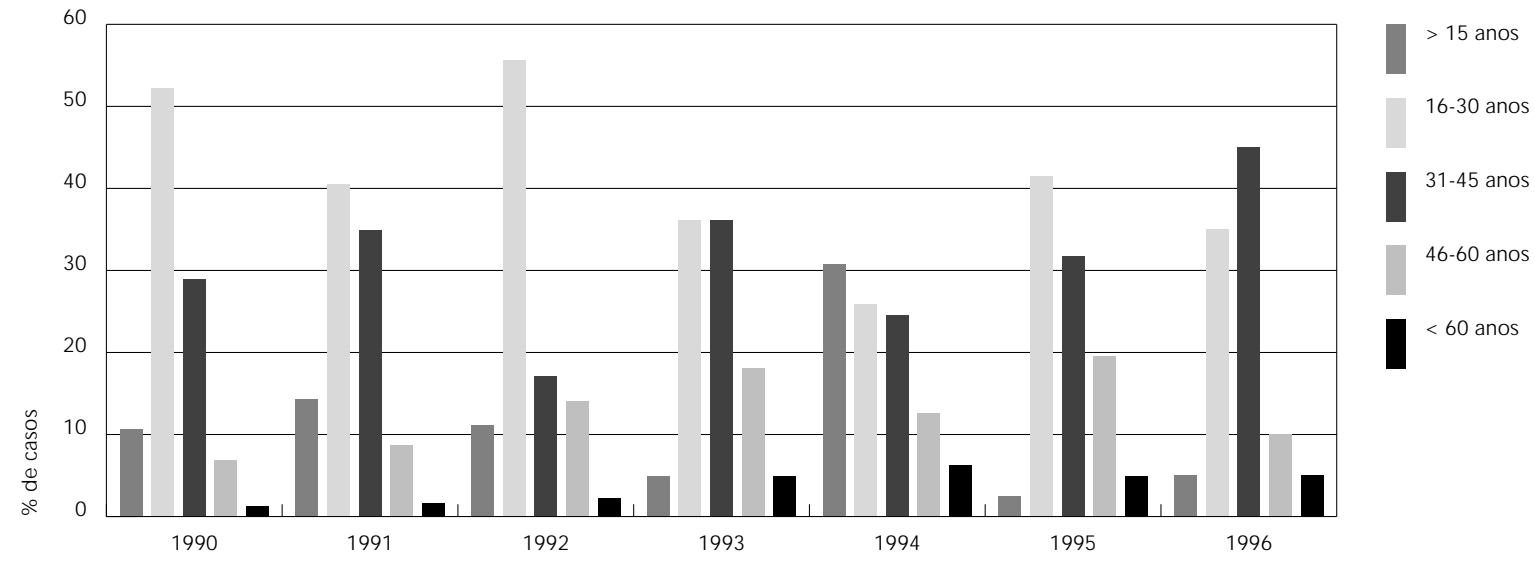

Fonte: MS/FNS/CO O R-M S/SEOPE, 1990-1997.

Figura 4

Malária - percentual de casos de malária, segundo a espécie de Plasmodium e ano registrados na Bacia do Alto Paraguai, Mato Grosso do Sul, no período de 1990 a 1996.

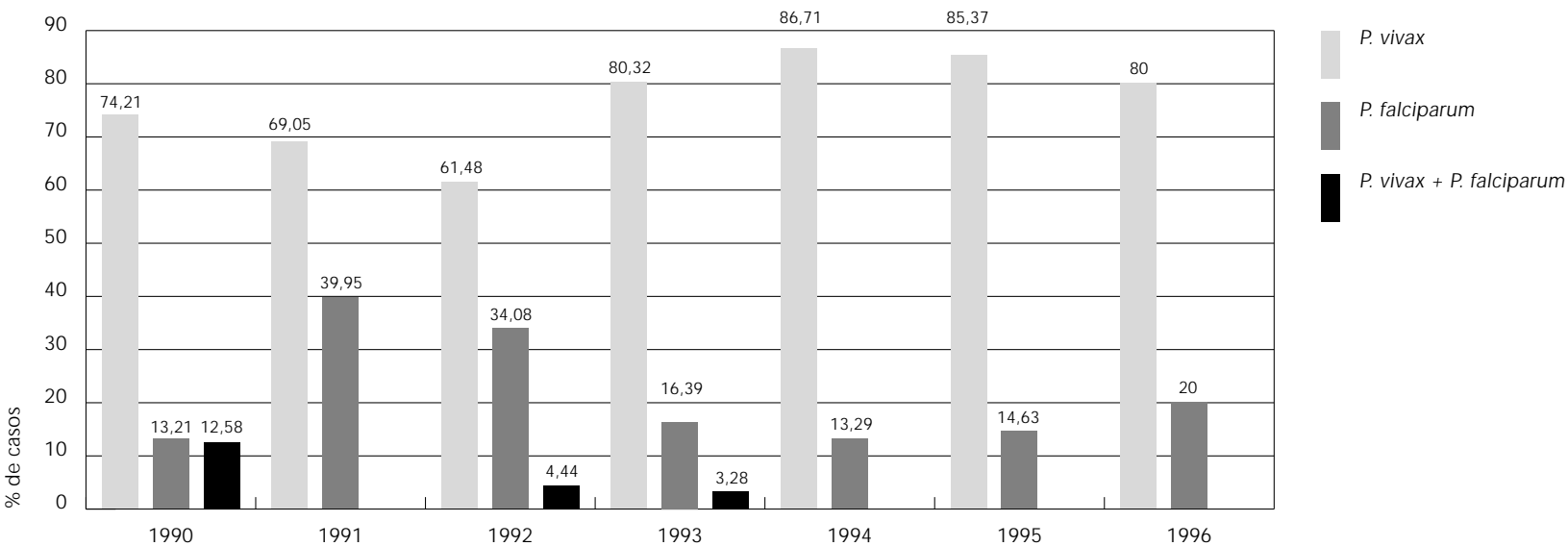

Fonte: MS/FNS/CO OR-MS/SEO PE, 1990-1997. 
rum foi responsável por $13,21 \%$ dos casos de 1990, aumentando para mais de 30\% em 1991 e 1992, voltando a declinar para índices inferiores a $20 \%$ nos anos subseqüentes. Os casos registrados em Mato Grosso do Sul por essa espécie também oscilaram de 19,26\% a 45,82\%, mas não ultrapassaram os percentuais de acometimento do Plasmodi um vivax. No Brasil, o Plasmodium falciparum predominou em dois períodos - 1966 a 1973 e 1984 a 1988 (Marques et al., 1994), ambos anteriores ao presente estudo. Há ainda relato de infecção na BAP/MS por ambas as espécies em três anos: 1990 (12,58\%), 1992 (4,44\%) e 1993 (3,28\%). Apesar de ocorrerem casos pelas duas espécies parasitárias em Mato Grosso do Sul em todos os anos da série histórica, os percentuais não ultrapassaram 2,10\% do total de casos (MS, 1990, 1991, 1992, 1993, 1994a, 1994b, 1994c, 1995a, 1995b, 1996a, 1996b), sendo também minoria no Brasil (Marques et al., 1994). Em 1994, dos 552.247 casos notificados no País, 64,55\% foram causados por Plasmodium vivax e Plasmodium falciparum, e 0,03\%, causados por Plasmodium malariae (MS, 1995a).

A grande maioria dos casos de malária registrados na Bacia do Alto Paraguai, Mato Grosso do Sul, no período em questão, é importada. Foram registrados dois casos induzidos, um em 1991 e outro em 1992. Ambos ocorreram por transmissão vertical, o primeiro em Coxim e o outro em Rio Verde de Mato Grosso. Casos autóctones foram registrados no período de
1991 a 1994, nos Municípios de Rochedo (1991), Pedro Gomes (1992) e Bonito (1993 e 1994) (Figura 5).

Em Mato Grosso do Sul também predominaram os casos importados, procedentes dos estados da região Norte do País, de Mato Grosso, de Goiás, e de dois países que fazem fronteira com o Estado - Paraguai e Bolívia (MS, 1990, 1993, 1996a).

Uma análise da situação da malária no Brasil no período de 1980 a 1993 aponta progressiva concentração de casos na Amazônia Legal e redução na Extra-Amazônia (Marques et al., 1994).

Com relação à educação em saúde, no Mato Grosso do Sul não existe uma programação definida de controle da malária, em razão do fato de os casos autóctones serem minoria no total investigado.

\section{Considerações finais}

Nos anos de estudo, os municípios que apresentaram maior número de casos de malária foram Coxim, Rio Verde de Mato Grosso, Bonito e Aquidauana, os dois primeiros pertencentes à microrregião de Alto Taquari e os outros, à microrregião de Bodoquena e Aquidauana, respectivamente. A faixa etária mais atingi da foi compreendida entre 16 e 45 anos. Houve predomínio do Plasmodium vivax no total de lâminas examinadas.

\section{Figura 5}

Malária - percentual de casos registrados na Bacia do Alto Paraguai/Mato Grosso do Sul, segundo a origem da infecção e ano, no período de 1990 a 1996.

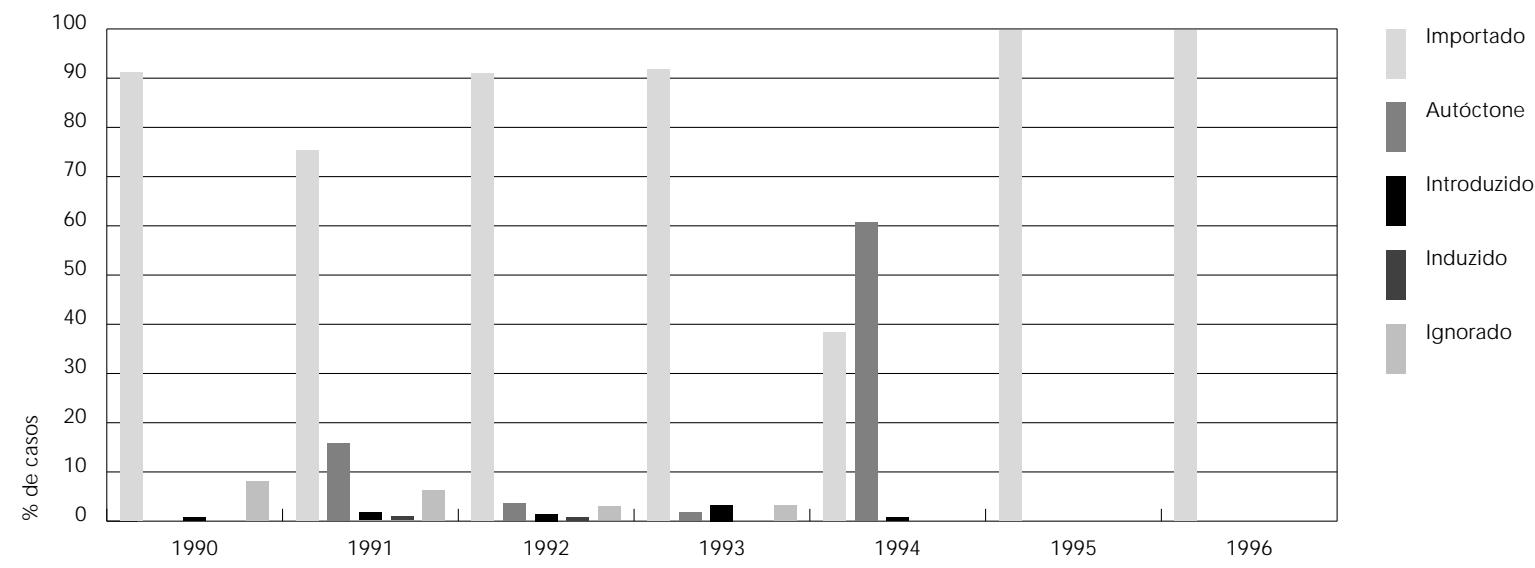

Fonte: MS/FNS/CO OR-MS/SEO PE, 1990-1997. 
Embora os casos autóctones de malária não sejam maioria, a doença constitui importante problema de saúde pública na Bacia do Alto Paraguai, em face da presença do vetor Anopheles (N.) darlingi e das correntes migratórias que se dirigem para a região.

Dois episódios confirmam essa afirmação. Primeiro, a epidemia ocorrida em Rochedo em 1991, com 22 casos, sendo vinte $(90,91 \%)$ autóctones, correspondendo a $100 \%$ dos autóctones do Estado naquele ano. Tal situação está associada ao contigente de trabalhadores procedentes da região Norte do País, contratados para suprir a necessidade de mão-de-obra pa-

\section{Referências}

KANOM ATA, E. H. N., 1994. Implantação de Focos Novos Ativos do 20 Grau, de Malária, em Mato Grosso do Sul esuas Repercussões no Controle de Endemia. Monografia apresentada ao Colegiado do Curso de Epidemiologia e Controle da Malária para a obtenção de título de Especialista. Cuiabá: Universidade Federal de Mato Grosso.

MARQUES, A. C. \& GUTIERREZ, H. C., 1994. Combate à malária no Brasil: evolução, situação atual e perspectivas. Revista da Sociedade Brasileira de Medicina Tropical, 27 (Sup. III):91-108.

MS (Ministério da Saúde), 1990. Relatório Anual do Programa de Controle da Malária. Campo Grande: Fundação Nacional de Saúde, Coordenação Regional.

MS (Ministério da Saúde), 1991. Relatório Anual do Programa de Controle da Malária. Campo Grande: Fundação Nacional de Saúde, Coordenação Regional.

MS (Ministério da Saúde), 1992. Relatório Anual do Programa de Controle da Malária. Campo Grande: Fundação Nacional de Saúde, Coordenação Regional.

MS (Ministério da Saúde), 1993. Relatório Anual do Programa de Controle da Malária. Campo Grande: Fundação Nacional de Saúde, Coordenação Regional.

MS (Ministério da Saúde), 1994a. Relatório Anual do Programa de Controle da Malária. Campo Grande: Fundação Nacional de Saúde, Coordenação Regional.

MS (Ministério da Saúde), 1994b. Manual deTerapêutica de Malária. Brasília: Fundação Nacional de Saúde. ra a cultura de arroz na região. Segundo, no ano de 1994, ocorre outra epidemia, agora no Município de Bonito, que registrou 93 casos, sendo 86 (92,47\%) autóctones, decorrente também de um fluxo migratório oriundo da região Norte, em conseqüência de notícias de suposta existência de metais preciosos na região.

É fundamental que a vigilância epidemiológica dessa doença seja fortalecida nos níveis municipal e estadual, para que os dados sejam regularmente coletados e anal isados a fim de permitir o acompanhamento e controle da patologia em Mato Grosso do Sul.
MS (Ministério da Saúde), 1994c. Guia deVigilância Epidemiológica. Brasília: Fundação Nacional de Saúde, Centro Nacional de Epidemiologia.

MS (Ministério da Saúde), 1995a. Informe EpidemioIógico do SUS. Brasília: Fundação Nacional de Saúde, Centro Nacional de Epidemiologia.

MS (Ministério da Saúde), 1995b. Relatório Anual do Programa de Controle da Malária. Campo Grande: Fundação Nacional de Saúde, Coordenação Regional.

MS (Ministério da Saúde), 1996a. Relatório Anual do Programa de Controle da Malária. Campo Grande: Fundação Nacional de Saúde, Coordenação Regional.

MS (Ministério da Saúde), 1996b. Informe Epidemiológico do SUS. Brasília: Fundação Nacional de Saúde, Centro Nacional de Epidemiologia.

OPAS (Organización Panamericana de la Salud), 1991. Estratificación epidemiológica de la malaria en la Región de las Américas. Boletín Epidemiológico. Washington: OPAS.

SUCEN (Superintendência de Controle de Endemias), 1988. Malária: Situação Atual, Diagnóstico, Tratamento e Controle. São Paulo: Sucen.

UFMS (Universidade Federal de Mato Grosso do Sul), 1996. Projeto de conservação da Bacia do Alto Paraguai. Relatório da Área de Saúde. Campo Grande: UFMS.

VERONEZI, R., 1983. Doenças Infecciosas e Parasitárias. 7ạ ed., Rio de Janeiro: Guanabara-Koogan. 